\title{
Insights into tribology from in situ nanoscale experiments
}

\author{
Tevis D.B. Jacobs ${ }^{1}$, Christian Greiner ${ }^{2}$, Kathryn J. Wahl ${ }^{3}$, and Robert W. Carpick ${ }^{4}$ \\ ${ }^{1}$ Department of Mechanical Engineering and Materials Science, University of Pittsburgh, USA \\ ${ }^{2}$ Christian Greiner, Karlsruhe Institute of Technology, and Institute for Applied Materials, Germany \\ ${ }^{3}$ Kathryn J. Wahl, Chemistry Division, Naval Research Laboratory, USA \\ ${ }^{4}$ Robert W. Carpick, Department of Mechanical Engineering and Applied Mechanics, University of \\ Pennsylvania, USA
}

The published version of this article can be found at: doi: $10.1557 / \mathrm{mrs} .2019 .122$ Full citation information is as follows:

Jacobs, T. D., Greiner, C., Wahl, K. J., \& Carpick, R. W. (2019). Insights into tribology from in situ nanoscale experiments. MRS Bulletin, 44(6), 478-486.

\section{Keywords:}

tribology; nanoscale; surface chemistry; atomic force microscopy; transmission electron microscopy

\begin{abstract}
:
Tribology - the study of contacting, sliding surfaces - seeks to explain the fundamental mechanisms underlying friction, adhesion, lubrication, and wear, and to apply this knowledge to technologies ranging from transportation and manufacturing to biomedicine and energy. Investigating the contact and sliding of materials is complicated by the fact that the interface is buried from view, inaccessible to conventional experimental tools. In situ investigations are thus critical in visualizing and identifying the underlying physical processes. This article presents key recent advances in the understanding of tribological phenomena made possible by in situ experiments at the nanoscale. Specifically, progress in three key areas is highlighted: (1) direct observation of physical processes in the sliding contact; (2) quantitative analysis of the synergistic action of sliding and chemical reactions (known as tribochemistry) that drives material removal; and (3) understanding the surface and subsurface deformations occurring during sliding of metals. The article also outlines emerging areas where in situ nanoscale investigations can answer critical tribological questions in the future.
\end{abstract}




\section{Introduction}

Tribology underlies the performance, safety, and reliability of nearly every mechanical system on land, at sea, and in space. The understanding and harnessing of tribological phenomena holds promise to address the $11 \%$ of annual energy consumption in transportation, utilities, and industrial applications lost due to friction and wear. ${ }^{1}$ This is in addition to the opportunities to save billions of dollars annually lost to downtime of industrial equipment, ${ }^{2}$ to eliminate billions of tons of $\mathrm{CO}_{2}$ emissions annually, ${ }^{3}$ and to significantly reduce human suffering caused by the failure of medical devices such as implants. ${ }^{4}$

Tribology depends on the physical, chemical, electrical, and system properties of the sliding materials, such as mechanical stiffness and strength, thermal and electrical conductance, hydrodynamic behavior, surface topography, material compatibility, temperature, sliding speed, and gas/fluid environment; in biological settings, a host of additional properties come in to play. Thus, the key parameters of interest such as the friction coefficient and wear rate are not material parameters, but rather, are system properties that vary with operational conditions. For newly engineered systems, or after a materials modification to an existing system, friction and wear performance are not currently predictable. Furthermore, the lack of direct observation of the sliding surfaces is a central obstacle to predicting performance and preventing failures. In situ techniques in tribology reveal the buried interfaces, as discussed in the 2008 issue of MRS Bulletin on the topic. ${ }^{5}$ The ensuing decade has brought significant advances both at the larger scales (e.g., Refs. 6-17) and at the nanoscale (e.g., Refs.18-22); the latter is the focus of the present article.

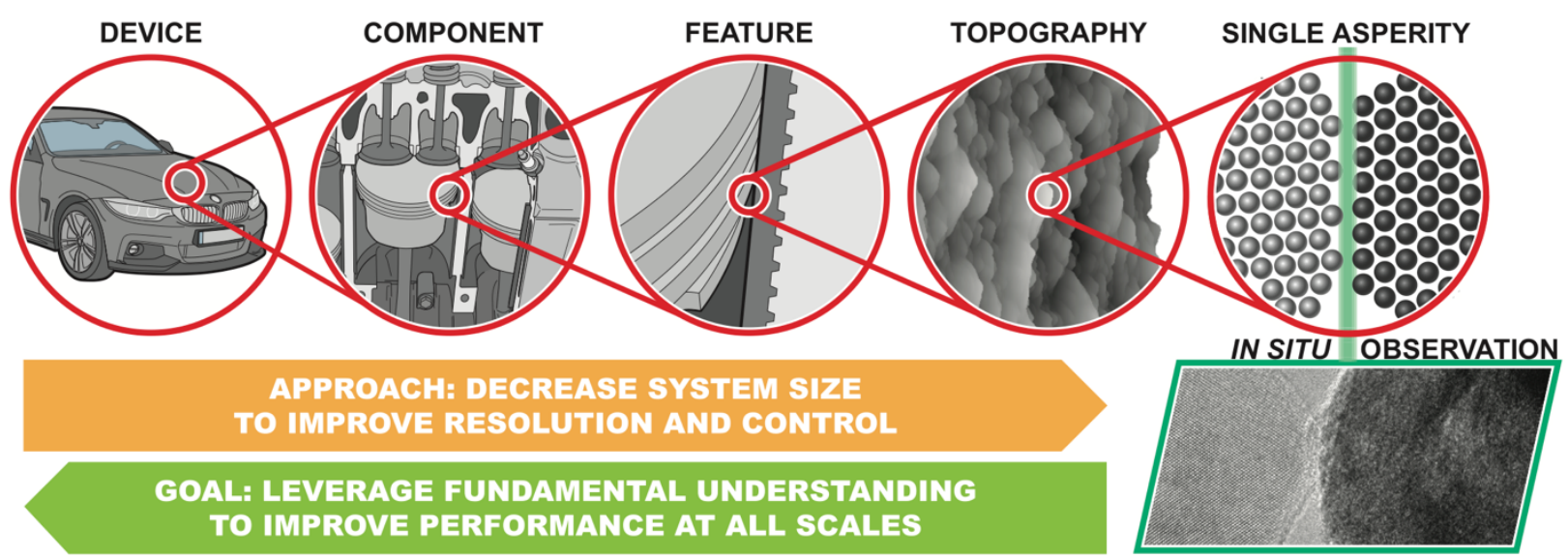

Figure 1. While tribological phenomena are complex and multiscale, fundamental understanding can be gained by reducing the system size to improve the control and measurement of local conditions. In situ nanotribology reveals the fundamental physical mechanisms and enables their systematic interrogation with the goal of informing design and optimization of larger-scale systems.

In situ nanotribology decreases the size scale of experiments to improve resolution and control over sliding conditions, with the goal of identifying and describing the underlying physical mechanisms (Figure 1). The fundamental understanding that is achieved from in situ nanoscale investigations can be generalized and harnessed at all length scales to improve tribological performance. Further, in situ 
experiments can be directly matched with atomistic simulations, enabling atomic-scale understanding of results. By establishing and quantifying the physical relationships that relate structure, processing, and properties, the goal is to enable the rational design of components, devices, and systems to improve tribological performance.

\section{Instrumentation for in situ nanotribology}

In situ nanotribology experiments include the use of microscopy or an analysis technique to characterize interfacial processes during sliding. One primary approach is to use specialty specimen holders to directly observe the tribological testing of nanoscale bodies with an electron microscope. ${ }^{23}$ This enables Ångström-scale resolution of morphology and surface topography. Simultaneously, the crystallographic structure, composition, and bonding chemistry can be characterized using analytical techniques such as nanobeam diffraction, energy dispersive x-ray spectroscopy (EDS), and electron energy loss spectroscopy (EELS). Another primary approach is to use atomic force microscopy (AFM) to perform both sliding and characterization. AFM enables precise control over the loading and sliding conditions, with simultaneous characterization of the surface's morphology and properties. ${ }^{24}$ The environment can be varied widely, covering vacuum, ambient, controlled atmospheres, and liquid, including biologically compatible environments.

Another type of investigation (termed quasi-in situ) interrupts sliding to analyze surfaces. This includes sliding an AFM probe for some distance before removing it for high-resolution imaging of the tip or sample, or sliding a pin on a disk followed by the sectioning of surfaces for electronmicroscopy analysis. Throughout this article, we discuss these quasi-in situ investigations as leading indicators, setting up the key scientific questions that will be answered by true in situ experiments.

\section{Revealing the buried interface: Key results and future directions Observations of novel phenomena}

In situ tribology experiments were first demonstrated in the 1960s by the famous tribologist Bowden ${ }^{25}$ who developed an in situ scanning electron microscope (SEM)-based indentation apparatus and studied small contacts of gold, copper, and aluminum; the in situ capability allowed Bowden and colleagues to observe that plastic deformation was negligible below a stress approaching the ideal strength of the metals tested; they then applied the instrumentation in a TEM. ${ }^{26}$ Kato and co-workers ${ }^{27}$ conducted in situ SEM-based wear studies of steel in 1988, directly observing a transition from plowing to wedge-forming to cutting as the degree of penetration increased. Shortly thereafter, Spence and co-workers developed a scanning tunneling microscope operating in situ inside a TEM, observing nanoscale compression of surfaces by the tip..$^{28,29}$

More recently, both commercial and custom-built TEM-based instruments have revealed a wide range of phenomena for several different material classes. For instance, in situ TEM investigation of contact between noble metals demonstrated "cold welding" of asperities upon contact, ${ }^{30,31}$ followed by liquidlike behavior during separation (discussed in more detail later). In the case of two-dimensional (layered) materials (e.g., graphite, $\mathrm{MoS}_{2}$ ), which are important solid lubricants, in situ TEM nanotribology has revealed rolling, ${ }^{32}$ exfoliation, ${ }^{33,34}$ and material transfer $^{35}$ during sliding. Another 
transformative aspect of in situ electron microscopy is the ability to reveal chemistry and bonding of the materials at the interface. For example, EELS analysis revealed changes in the hybridization state of carbon-based materials during in situ contact. Merkle and co-workers ${ }^{36}$ demonstrated a slidinginduced increase in the $s p^{2}$-to-sp $p^{3}$ ratio of diamond-like carbon; conversely, Janei and co-workers ${ }^{37}$ demonstrated a decrease in $s p^{2}$ content of soot particles after in situ compression.

Other recent work has leveraged the ability to work in biocompatible fluids, where in situ work has demonstrated links between tribological behavior and biological response. ${ }^{38}$ This includes the effects of shear forces on the growth and proliferation of cells, as elucidated by approaches such as the direct observation of contact between a soft hydrogel cap and a layer of corneal epithelial cells imaged via fluorescence microscopy, ${ }^{17}$ or the measurement of friction and shear force-induced inflammation of cells in vitro. ${ }^{39}$

These examples and many others demonstrate the power of in situ approaches to tribology. We next discuss selected studies in more detail that have advanced knowledge of tribology through direct observations at the nanoscale, and have uncovered or confirmed specific physical mechanisms underlying processes related to contact, adhesion, friction, and wear at all length scales.

\section{The role of tribochemistry in material removal}

Tribochemistry is the acceleration of chemical reactions at surfaces by sliding. Tribochemistry builds on the well-established field of mechanochemistry, which describes how mechanical forces can alter the kinetics of chemical reactions. However, the sliding action adds further complexity in the form of spatially and temporally varying loads, evolving surface morphology and chemistry, and the transport of reactants and products into and out of the contact region. In many applications, material removal by tribochemistry plays a primary role in sliding wear. A common theme that has emerged is the importance of thermally and tribologically activated chemical processes affecting wear. In situ investigations provide a way to understand, and even potentially predict and control, these wear processes.

\section{Tribochemical wear in silicon and carbon-based materials}

Significant wear and surface modification can occur in covalently bonded materials, even well below the fracture stress. ${ }^{40}$ Quasi-in situ investigations using atomic force microscopy have demonstrated that material removal could be modeled using reaction rate theory. ${ }^{41-45}$ Gotsmann and co-workers used high-speed AFM to study the wear of silicon ${ }^{43}$ and carbon $^{44}$ nanoprobes with periodic adhesion measurements (as an indirect measure of probe radius) and ex situ electron microscopy (as a direct measure). They demonstrated that the wear was gradual, and could be described by combining reaction rate theory with an empirical model for friction stress, and the conical geometry of the probe. ${ }^{43}$

In situ nanoscale investigations have further advanced the understanding of tribochemical material removal. TEM observations of silicon AFM probes sliding on diamond (Figure 2a) demonstrated Angström-scale recession of the silicon nanoprobe while the subsurface crystallography was 
maintained. ${ }^{18}$ Significant material removal occurred in the absence of fracture, plasticity (i.e., subsurface defects or permanent shape change), or observable debris (Figure $2 b$ ). The improved spatial and temporal resolution of the in situ TEM testing enabled the characterization of instantaneous tip shape and the quantification of the rate of atom removal. By combining the asperity shape with subnanonewton force resolution, the local and instantaneous mean contact stress could be computed. Together, these measurements provided direct evidence that sliding wear occurred through surface reactions with an Arrhenius dependence on local stress, (Figure 2c) where the atomic reaction rate $\Gamma$ (with units of $\mathrm{s}^{-1}$ ) is given by: ${ }^{45}$

$$
\Gamma=\Gamma_{0} \exp \left(-\frac{\Delta G_{a c t}}{k_{B} T}\right)
$$

where the prefactor $\Gamma_{0}$ includes the effective attempt frequency of the reaction, which is related to atomic vibration frequencies, $\Delta G_{a c t}$ is the Gibbs free energy of activation for the rate-limiting reaction in the process, $k_{B}$ is Boltzmann's constant, and $T$ is the absolute temperature. The fit to the data assumes that $\Delta G_{a c t}$ is influenced by stress according to:

$$
\Delta G_{a c t}=\Delta U_{a c t}-\sigma \Delta V,
$$

where $\Delta U_{a c t}$ is the internal activation energy (energy barrier in the absence of stress), $\sigma$ is the mean value of the stress component affecting the activation barrier, and $\Delta V$ is the activation volume. In this case, $\sigma$ was taken as the compressive stress (which assists in covalent bond formation across the interface); however, because the interfacial shear stress depends on the compressive pressure, it is difficult to distinguish which stress is "activating" in a given situation.
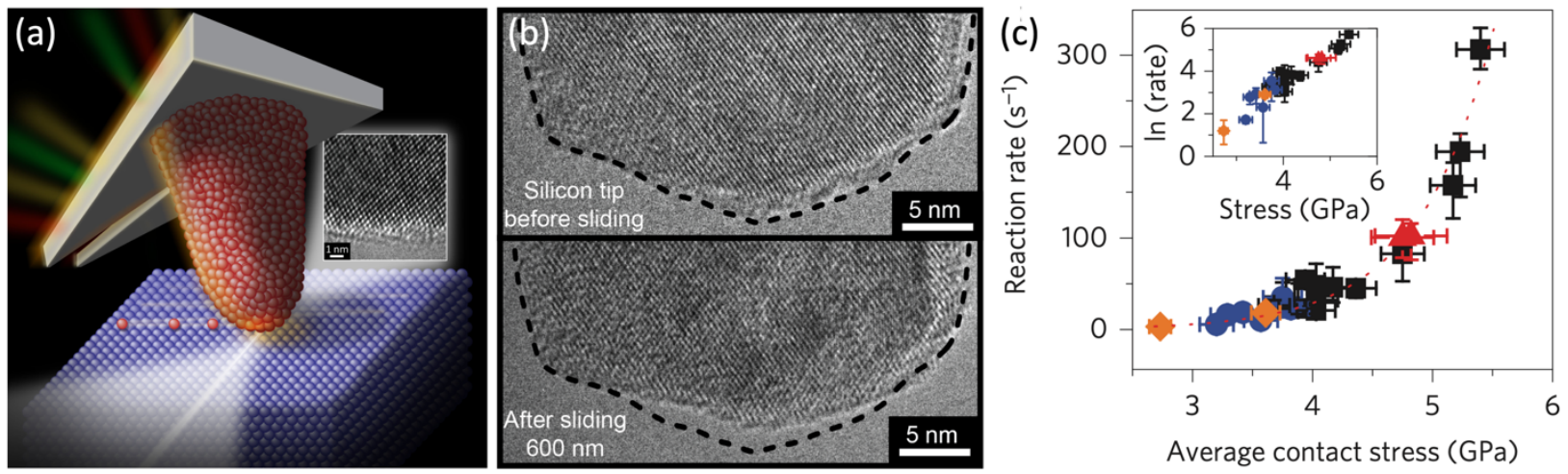

Figure 2. The sliding of a silicon atomic force microscope probe on diamond in a transmission electron microscope (a) enables characterization of the evolving shape and structure during wear (b). ${ }^{46}$ Combining the wear data with real-time load measurements (c) enables the direct demonstration of reaction rate theory (Equation 1) and the extraction of activation parameters for low-load wear of silicon. ${ }^{18}$ The inset in (c) shows that the data collapses to a straight line on a log-linear plot.

AFM wear experiments with in situ tip-based heating were performed on functionalized graphene surfaces (Figure 3) to further interrogate the stress-dependent kinetics of bond breaking and the effect of temperature on bond-scission dynamics. ${ }^{19,47}$ Real-time friction measurements were used as an in 
situ measurement of molecular-scale material removal, and the heated AFM probes directly applied temperature ramps to examine temperature-dependent rates of material loss ${ }^{47}$ In contrast to prior experiments that assumed thermal activation, first-order reaction kinetics were used to verify an Arrhenius dependence of the material removal rate on inverse temperature. Additionally, by controlling applied load and contact time, the authors were able to measure the different kinetics of oxygen-, fluorine-, and hydrogen-functionalized graphene. ${ }^{19}$

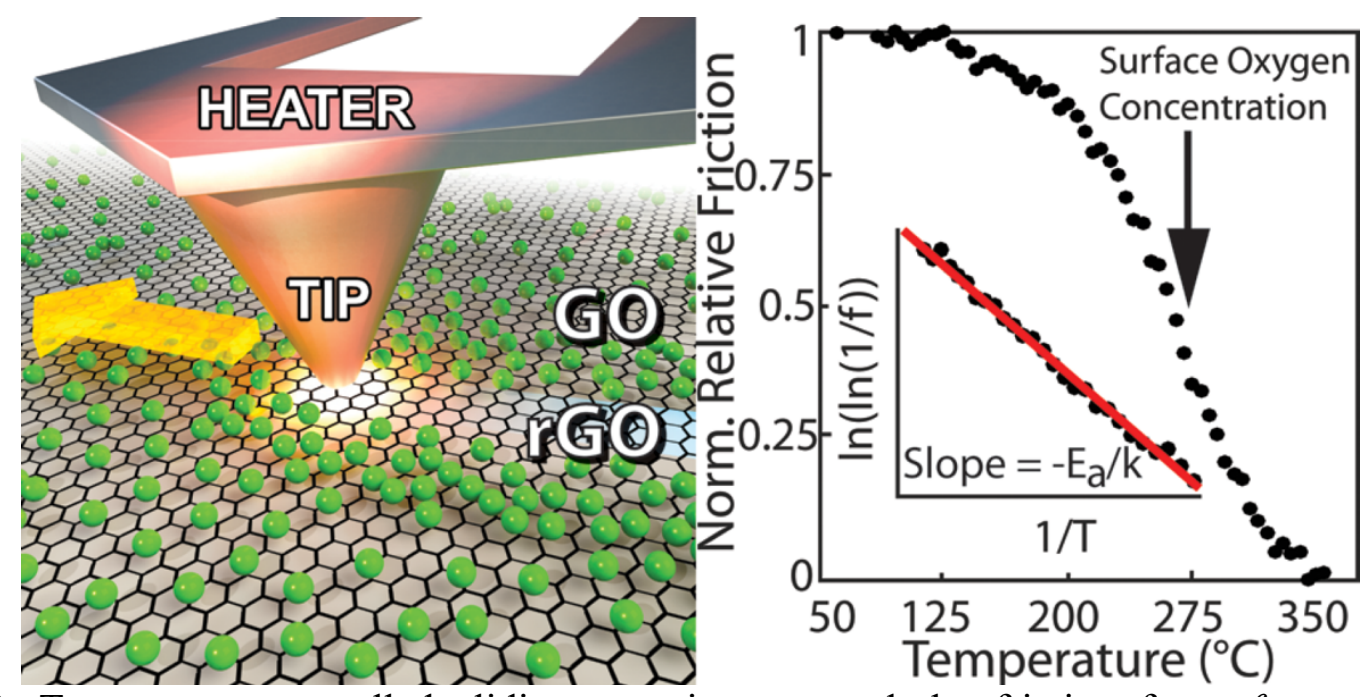

Figure 3. Temperature-controlled sliding experiments used the friction force $f$ as a real-time measurement of surface coverage of functional groups on graphene. Here, the removal of oxygen groups from graphene oxide (GO) produces reduced graphene oxide ( $\mathrm{rGO})$. By varying the temperature, the thermal activation was directly confirmed and the activation parameters of bond scission were extracted. In this graphic, $E_{\mathrm{a}}$ is activation energy and $k$ is Boltzmann's constant. Reprinted with permission from Reference 47. (C) 2017 American Chemical Society.

The importance of these stress-controlled bonding reactions were also demonstrated for adhesive contacts even in the absence of sliding. In situ TEM experiments of amorphous carbon tips in contact with diamond showed gradual material removal, and fluctuations in the adhesion force due to covalent bond formation during contact. ${ }^{48}$ Finally, interrupted (quasi-in situ) imaging of amorphous carbon tips in sliding contact with diamond revealed a load-dependent transition from an exponential dependence on stress (the Arrhenius-like behavior described in Eqs. 1-2) to the linear dependence described by the Archard equation for wear. ${ }^{49}$

Taken together, these recent in situ nanoscale investigations established stress-modified thermally activated bond-breaking as the key framework for describing low-load wear behavior of covalent materials. The predictive power of this framework, and its limits of applicability, are still being actively explored. 


\section{Tribochemical buildup and removal of antiwear additives}

Industrial lubricants contain a substantial fraction (up to ca. $10 \mathrm{wt} \%$ ) of additives for important functions, including reducing boundary friction, controlling viscosity, and reducing wear. Particularly important is the family of zinc dialkyldithiolphosphate (ZDDP) additives, which are used in every commercial lubricant for internal combustion engines. These tribochemically active molecules reduce wear and corrosion by forming thin protective films ("tribofilms") through adsorption and confinement at contacting asperities, followed by force-induced dissociation and subsequent reactions (Figure 4). ${ }^{20,50,51}$ ZDDPs are inexpensive and highly effective, but contain sulfur and phosphorous that poison catalytic converters, thereby increasing harmful emissions. The automotive industry has for decades sought a suitable replacement but has not yet succeeded. One reason is that the underlying tribochemical behavior at the asperity level was not well understood. Macroscopic, ex situ, and quasiin situ studies ${ }^{51-55}$ have elucidated the structure and composition of these films; experiments have shown that compressive ${ }^{56}$ and shear $^{50}$ forces are crucial for film formation, but insights to explain the tribofilms' graded structure and self-limiting growth (at approximately 100-nm thickness) are longstanding challenges.

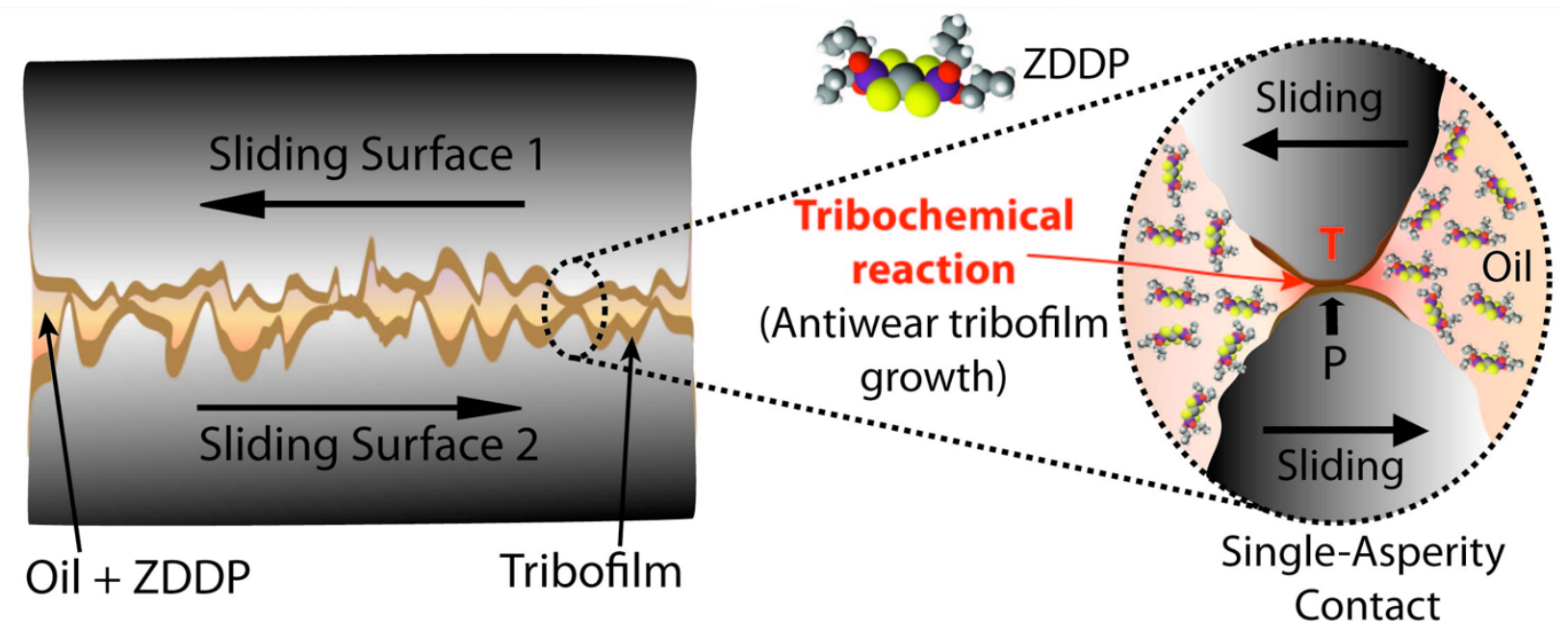

Figure 4. A challenge in understanding force-dependent reactions is that the contacting surfaces are not atomically smooth, with energy dissipated largely at nanoscale asperity-asperity contacts. This affects the reactions of molecules such as zinc dialkyldithiolphosphate that undergo shear-assisted reactions at the single-asperity scale forming surface-bound films. ${ }^{20}$ Image courtesy of N.N. Gosvami, Indian Institute of Technology, Delhi.

Gosvami et al. recently developed an AFM method where tribofilms are created in situ while simultaneously probing nanoscale properties including morphology, friction, and wear (Figure 5a). ${ }^{57}$ A single-asperity contact is formed between an AFM tip and a flat sample submerged in a conventional AFM liquid cell containing the lubricant. Alternatively, multi-asperity contacts can be formed by using a rough microscale colloidal tip. ${ }^{57}$ Applied force, sliding velocity, and temperature can be varied, and thus nucleation and growth of tribofilms are mapped in real-time against shear rate, stress, and temperature. Gosvami et al. ${ }^{20}$ heated the ZDDP-containing polyalphaolefin oil to $80-140^{\circ} \mathrm{C}$ during 
sliding experiments, which were performed for a range of normal loads. Sliding-induced ZDDP tribofilms grew at a rate that was well-described by Equation 1 (Figure 5b). Again, $\sigma$ was assumed to be the initial compressive contact pressure, but shear stress could in fact be controlling the reaction, as shown in macroscopic studies by Zhang and Spikes; ${ }^{50}$ the model holds if the shear stress is proportional to the normal stress. Fitting of Equations (1) and (2) (Figure 5b-c) gave values of $\Delta U_{a c t}=$ $0.8 \pm 0.2 \mathrm{eV}$ and $\Delta V=3.8 \pm 1.2 \AA^{3}$, consistent with a molecular-scale process.
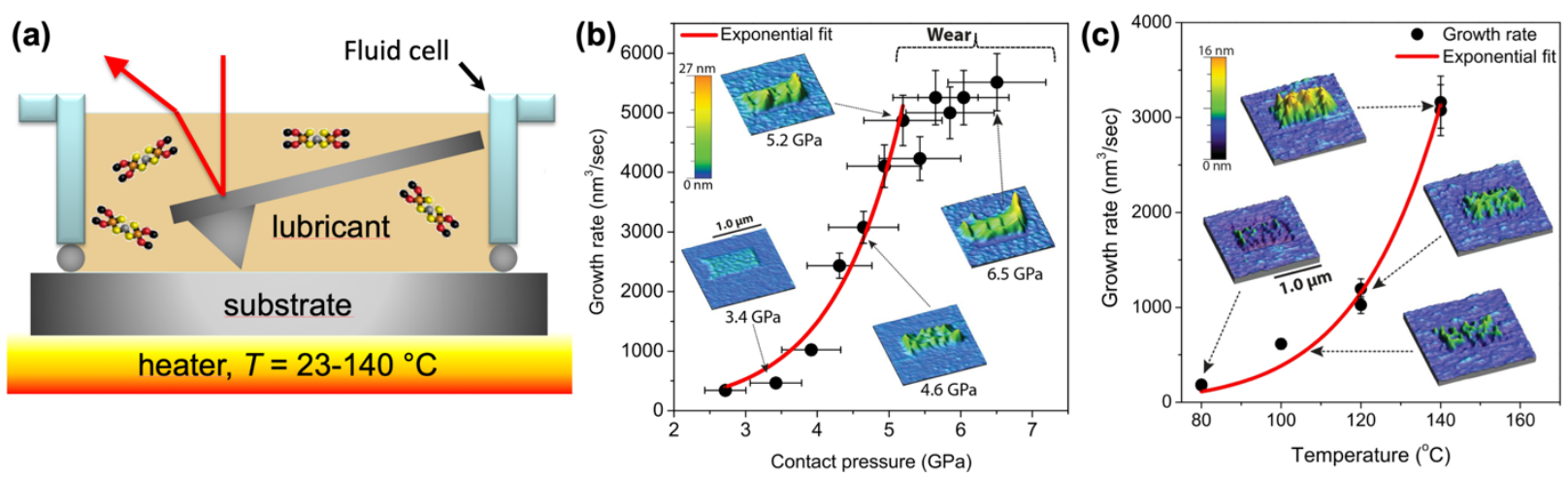

Figure 5. (a) Schematic of the atomic force microscopy setup immersed in a lubricant (zinc dialkyldithiolphosphate [ZDDP] additive molecules schematically shown) used for in situ tribofilm formation. (b) ZDDP tribofilm growth rate versus mean applied normal contact stress at constant temperature, fit with an exponential (stress-activated) function (Equations 1 and 2). (c) Tribofilm growth rate versus temperature at constant normal stress, also fit with an exponential function, Insets to (b) and (c): log of growth rate versus stress and temperature, respectively. (b, c) Reprinted with permission from Reference 20. (C) 2015 AAAS.

Stress-activated growth explains why ZDDP-derived tribofilms have a graded structure and a selflimiting thickness. Reduced contact pressure — resulting from the tribofilm's low modulus_limits the degree of cross-linking and other tribofilm-forming reactions, resulting in a graded structure with progressively less cross-linking and a lower modulus, thus further reducing the contact pressure. The growth rate thus reduces and eventually tapers off as the film grows, a sort of "cushioning" effect, which is supported by a recent asperity-based kinetic model.${ }^{58}$ Dorgham et al. ${ }^{59}$ further demonstrated the versatility of this method, when they compared ZDDP with an ashless (metal-free) DDP, finding that the reaction order is different, indicating that the tribochemical reaction pathways depend significantly on the molecule's structure. This in situ method has also been used to form and study tribofilms derived from solid $\mathrm{ZrO}_{2}$ nanoparticle additives in oils, ${ }^{60}$ and applied to form patterned tribofilms on surfaces, termed "nanotribological printing." 61

\section{Tribocorrosion}

The synergism between chemistry and material removal is even more severe in corrosive environments, including biomedical implants, ${ }^{62}$ nuclear power plants, ${ }^{63}$ and marine environments. ${ }^{64}$ Even in corrosion-resistant alloys, sliding action causes repetitive loss of protective surface films, resulting in the loss of metal ions to repassivate the worn surface and thereby accelerating material 
degradation. ${ }^{65}$ Corrosion-resistant metals typically contain costly alloying elements such as $\mathrm{Ni}$ and Co, and can have complex microstructures with carbide or nitride inclusions. Besides solution chemistry and sliding contact conditions, the wear-corrosion synergy is controlled by factors such as composition, applied potential, applied or residual stresses, and fatigue resistance., ${ }^{966-68}$ The mechanisms by which local material properties influence tribocorrosion processes are underexplored.

The state-of-the-art involves breakthroughs from quasi-in situ investigations. Malayoglu and Neville ${ }^{69}$ used examination of worn surfaces by AFM to show that the preferential removal of Co-rich matrix material was more pronounced on cast versus hot isostatically pressed (HIPed) CoCr alloys. Wang et al. ${ }^{70}$ revealed that preferential dissolution at the boundary region between carbides and the CoCrMo matrix accelerates abrasive wear in medical implant alloys. Shockley et al ${ }^{16}$ demonstrated, for an aged duplex stainless steel, that sliding wear initiated runaway pitting corrosion in susceptible phases, which did not occur in the absence of sliding.

These advances motivate locally probing fundamental material processes of sliding wear in corrosive environments. For instance, while repassivation kinetics is modeled in its simplest form using Faraday's Law ${ }^{65}$ and has been explored for microscale scratching by diamond tips ${ }^{63}$ the periodic removal and repassivation of oxide films that occurs in tribological sliding is not well understood. Further, current tribocorrosion modeling relies on the assumption of full oxide removal, ${ }^{65}$ while realworld multi-asperity contacts may experience only partial removal of the oxide. Fully in situ nanoscale investigations based on electrochemical scanning probe microscopy represent a promising pathway for enriching our understanding of the local processes in tribocorrosion.

\section{Surface and subsurface processes in metals}

The majority of tribologically loaded engineering components are metals, including noble metals for specialty applications and oxide-forming metals for general use. The understanding of deformation and energy dissipation in these metals is being significantly advanced by in situ and quasi-in situ investigations.

\section{Surface adhesion (or cold-welding) of metal contacts}

Adhesive wear, often referred to as galling, typically results from the combined action of friction and adhesion on sliding pairs, and can be a source of significant energy dissipation and material removal or degradation. The spontaneous bonding of metals has been extensively studied using the approach of mechanically controllable break junctions, where wires are pulled to breaking in a high-vacuum environment and then brought back into contact. Many of these contact-and-separation experiments have been quasi-in situ, where electrical current was recorded as an indirect measure of junction size; these have demonstrated the liquid-like separation and reforming of the contact (sometimes referred to as cold welding). ${ }^{71-73}$ As mentioned earlier, fully in situ nanoscale experiments of nanoscale junctions $\mathrm{s}^{30,31}$ established that many noble-metal contacts separate in a progressively thinning ligament that culminates, in some cases, in a single-atom chain before separation. These results have been 
confirmed for tribologically relevant contacts, including gold and silver. ${ }^{21,74-77}$ This liquid-like behavior has even been observed in nonmetallic contacts of aluminum oxide ${ }^{78}$ and silicon carbide. ${ }^{79}$ The fundamental understanding of this liquid-like contact behavior has been advanced using in situ nanoscale investigations. Using contact and sliding tests in the TEM on silver, ${ }^{80}$ gold, ${ }^{81}$ and oxide-free $\operatorname{tin}^{22}$ nanocontacts, it was shown that this deformation was accommodated through the motion of surface steps in the material surrounding the contact. After separation, asperities regained a similar resting shape regardless of the amount of deformation induced. This indicated deformation that was mediated by surface diffusion (Figure 6a-b), analogous to Coble creep, where time-dependent deformation in load-bearing bulk materials occurs via atomic diffusion along grain boundaries.
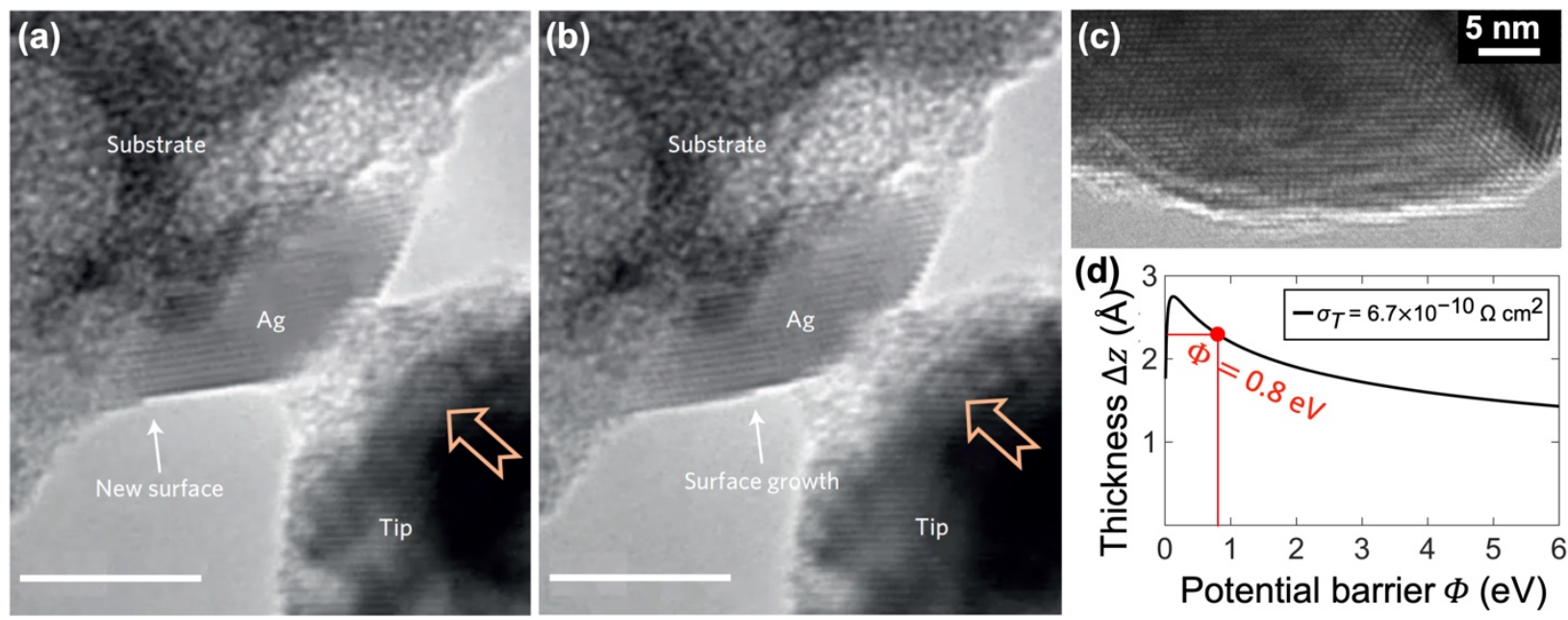

Figure 6. In situ transmission electron microscopy compression of silver contacts shows ${ }^{80}$ that deformation is accommodated through the motion of surface steps $(\mathrm{a}, \mathrm{b})$. (The scale bars are $5 \mathrm{~nm}$ in length.) However, subsurface defect-based plasticity is observed ${ }^{85}$ in platinum nanocontacts (c), and real-time electrical measurements ${ }^{86}$ are consistent with the presence of atomic-scale surface layers $(\mathrm{d})$, which may disrupt surface diffusion. (a,b) were reproduced with permission from Reference 80, (C) 2014 Nature Publishing Group. (c, d) were reproduced with permission from Reference 86, (C) 2018 IOP Publishing.

Tin contacts were described using a Coble-creep model that relates the stress $\sigma$ to the strain-rate $\dot{\varepsilon}:{ }^{22}$

$$
\dot{\mathrm{E}}=K \frac{\delta_{S} D_{S} \Omega}{D^{3} k_{B} T} \sigma
$$

where $D$ is the characteristic dimension (e.g., diameter of the asperity) and $K$ is a dimensionless constant. The material properties are described by surface diffusivity $D_{S}$, nominal surface layer thickness $\delta_{S}$, and atomic volume $\Omega$. Li and co-workers combined in situ nanotribology experiments with simulations to delineate regimes where deformation will be liquid-like (what the authors call diffusive plasticity ${ }^{82}$ ) or dislocation-mediated (termed displacive plasticity ${ }^{82}$ ). Both processes can occur simultaneously, ${ }^{83}$ but a criterion based on MD simulations and in situ nanoscale experiments determines which process will dominate. ${ }^{84}$ In dislocation-based plasticity, "smaller is stronger," as 
expressed by the Hall-Petch relationship, $\sigma_{\text {flow }}=\sigma_{0}+k D^{-1 / 2}$, where $\sigma_{0}$ and $k$ are constants. In contrast, in surface diffusion-dominated deformation with a specific material, temperature, and flow rate, the flow stress is governed by $\sigma_{\text {flow }}=\sigma_{0}+k D^{3}$, which indicates that "smaller is much weaker."

Separate in situ TEM adhesion experiments demonstrated the critical role that surface chemistry plays in the deformation of platinum nanocontacts. Liquid-like diffusive behavior is not observed in selfmated platinum nanocontacts, ${ }^{85}$ likely due to the presence of surface monolayers of oxygen or carbonaceous material (Figure 6c-d), ${ }^{86}$ which is consistent with atomistic simulations. ${ }^{87}$ These surface layers prevent the motion of the surface steps that are required for diffusive plasticity. Surprisingly, these surface layers are not removed by mechanical means, even with loading and sliding in vacuum. ${ }^{86}$

\section{Subsurface dislocation processes in metal contacts}

Dislocation-mediated processes in the subsurface region of a contact are critical for understanding deformation and energy dissipation in metals in sliding contact. ${ }^{88}$ The movement and self-organization of dislocations leads to a dynamic and complex subsurface microstructure. ${ }^{89,90}$ The structureproperties relationship of a tribological contact was recently described as a feedback loop between grain size, friction, and surface stresses. ${ }^{91}$ This suggests opportunities to tailor alloys for tribological applications; however, doing so requires revealing the mechanisms governing these microstructural processes. In situ TEM experiments of moving tribological contacts represent an important opportunity, yet such experiments are very challenging, both in instrumentation and in application to real-world conditions. For instance, in situ TEM requires electron transparency and thus ultrathin widths, yet these small dimensions introduce image forces from the free surfaces and other thin-film effects that strongly affect results. ${ }^{92}$
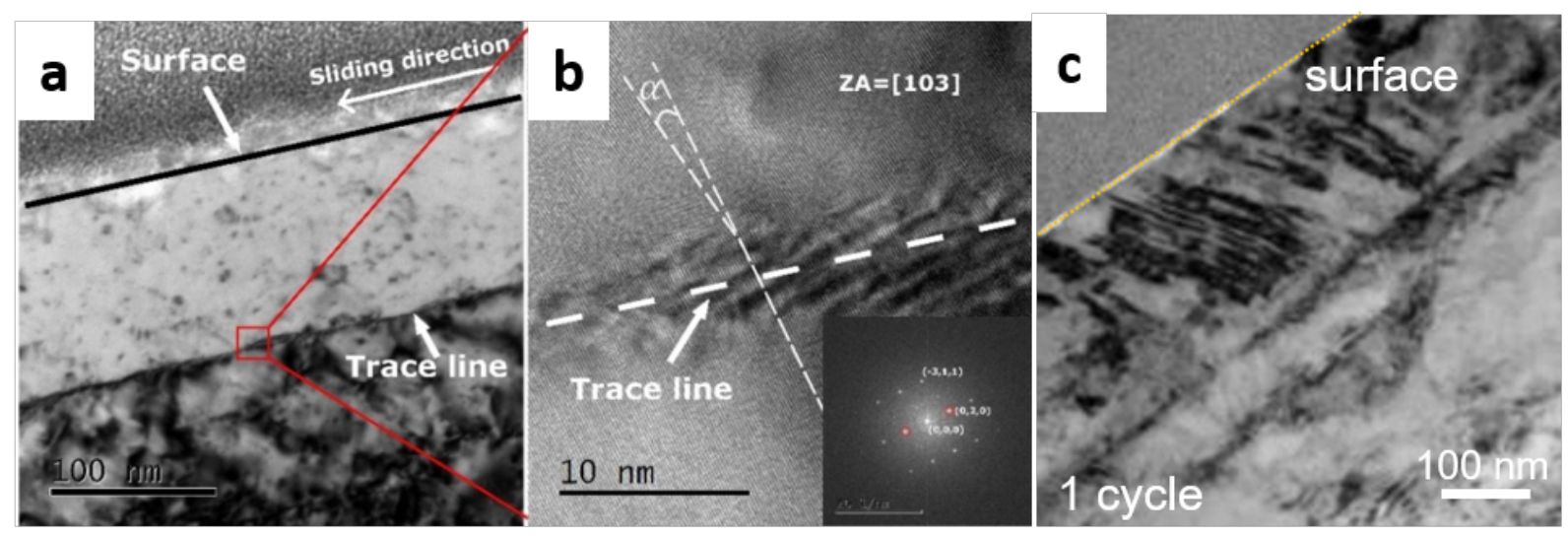

Figure 7. Dislocation self-organization during the very early stages of sliding leads to a horizontal small-angle grain-boundary-like feature, which can be observed using transmission electron microscopy after only a single sliding event $(a, b)$. The foil was tilted to a [103] zone axis (ZA).$^{93}$ The line-like feature was found to be a boundary separating regions that accommodate shear differently, with dislocation motion dominant below the line and twinning dominant above, as shown in (c). ${ }^{95}$ 
Critical advances have been established using quasi-in situ tribological experiments, with highresolution TEM analysis of the subsurface structure. Experiments on annealed copper revealed that after only one mild pass of a counter body, dislocations self-organized and formed a horizontal dislocation feature similar to a small-angle grain boundary $150 \mathrm{~nm}$ under the surface (Figure 7a-b). ${ }^{93}$ This feature was the precursor to microstructural discontinuities observed upon further sliding ${ }^{15,94}$ and also separated the regions where deformation occurred primarily through twinning or through dislocation motion (Figure 7c). ${ }^{93,95}$ With increasing sliding distance, large quantities of dislocations are emitted from and through the sliding contact to a depth of several micrometers. Dislocations selforganized, first into networks of geometrically necessary dislocations, then into sub-grains, and ultimately into a fine-grained "tribo-layer." $96-98$ This method of interrupted sliding on non-noble metals has also helped to understand how pearlitic steels accommodate a tribological strain through a sequence of different processes ${ }^{99}$ and has shed light on tribologically induced oxidation. ${ }^{100}$

At the same time, this current state of the art is not satisfactory. Postmortem analyses inherently allow different interpretations for how the observed structures have been achieved. For instance, MD simulations ${ }^{101}$ have suggested the importance of the Bauschinger effect, where the back-stresses of dislocations that are induced during loading in the forward direction serve to aid the activation of dislocation slip in the reverse direction. Complete understanding of real-time processes requires in situ nanoscale analysis. A recent first step in this direction linked in situ TEM with MD to demonstrate that the contact area in a metal nanocontact can be up to $160 \%$ larger than predicted by classical continuum theories due to dislocation nucleation and motion, even for contacts without irreversible shape change. ${ }^{85}$ These results demonstrate the need to closely consider dislocation activity even in pseudoelastic contacts, and more broadly, show the potential for future in situ TEM testing of subsurface deformation of metal nanocontacts.

\section{Summary and outlook}

In situ nanotribology has enabled breakthroughs in the understanding of fundamental mechanisms in tribology, and it represents a powerful platform for current and future advances in the field. This progress has been facilitated by advancements in electron microscopy, where aberration-correction equipment has enabled single-atom resolution and also larger physical pole-piece gaps to accommodate an increasing range of in situ tools. In situ tribology investigations have only just begun to leverage the analytical capabilities of electron microscopy to analyze the chemistry and bonding at the interface. The proliferation of environmental TEM equipment is ideally suited for tribological experiments - by enabling the introduction of humid or gas environments during testing, experiments can more accurately reflect real-world conditions in many tribological contacts. However, to take full advantage of these TEM advances, further improvements are required for the in situ TEM test platforms. Key limitations of existing test platforms include: vibration and drift, which limit image quality and precision of movement; limited tilt range, which prevents 3D characterization using tomographic techniques; and the use of complex or custom fixturing, which significantly limits throughput and thus sample size and statistical significance of results. There are promising approaches to address some of these limitations, including integrating the test surfaces directly into a 
microelectromechanical systems-based chip to improve stability; ${ }^{102}$ however, further progress is needed, especially using commercial in situ TEM testers, to fully leverage the advantages of modern TEM.

There is still much work to be done to apply the mechanisms revealed using in situ nanoscale testing to bulk conditions. For example, friction coefficients and wear rates for the same material can differ by more than an order of magnitude when measured at nanoscopic and macroscopic dimensions. ${ }^{103}$ These inconsistencies reflect important differences in conditions, such as: wearless sliding in an AFM as compared to wear-inducing sliding in bearings; or dry in situ TEM tests as compared to lubricated engine conditions; or an adhesive single asperity under gigapascals of contact stress as compared to a (nominally) flat contact under apparent stresses that are orders of magnitude lower, and where contact occurs at many asperities simultaneously. In many cases, the underlying physical processes are related across all scales of tribology, but differences in conditions can modify the mechanisms and their kinetics and energetics.

Three key needs stand out for further scientific advancements of tribology in the future. First, true in situ investigations are needed in cases where the current state-of-the-art is quasi-in situ investigation. For example, the quasi-in situ investigation of plasticity in a sliding contact must be advanced through the real-time observation of dislocation creation and self-organization. Second, while significant progress has been made in single-asperity experiments and atomistic modeling of nanoscale contacts, the field is still lacking the multi-scale modeling and combined multi-resolution experiments needed to bridge length and time scales and to directly apply fundamental insights to macroscale components. This includes work on biological systems, where many opportunities abound. ${ }^{38}$ Third, significant improvements are needed in the description and understanding of surface topography and its effect on properties. Scaling-up of knowledge requires not just an increase in the size of the contacting components, but also a more complete understanding of the statistical distribution and behavior of roughness at all scales. Continued advancement in these three areas will enable the ultimate goal of rational, science-based improvement of the tribological performance of sliding components in realworld devices.

\section{Acknowledgments}

T.D.B.J. acknowledges support from the National Science Foundation through Award CMMI1536800. C.G. acknowledges funding by the German Research Foundation under Project GR 4174/1 and by the European Research Council (ERC) under Grant No. 771237, TriboKey. K.J.W. acknowledges support from the Base Program at the Naval Research Laboratory via the Office of Naval Research. R.W.C. acknowledges support from the National Science Foundation through Awards CMMI-1728360 and CMMI-1761874, and from AFOSR/AOARD though Award FA238618-1-4083. The authors wish to thank W.G. Sawyer, N.N. Gosvami, R. Bernal, F. Dassenoy, and J. Felts for useful discussion. 


\section{References}

1. R.W. Carpick, A. Jackson, W.G. Sawyer, N. Argibay, P. Lee, A. Pachon, R.M. Gresham, Tribol. Lubr. Technol. 72, 44 (2016).

2. H.P. Jost, Wear 136, 1 (1990).

3. K. Holmberg, A. Erdemir, Friction 5, 263 (2017).

4. S. Affatato, M. Spinelli, M. Zavalloni, C. Mazzega-Fabbro, M. Viceconti, Med. Eng. Phys. 30, 1305 (2008).

5. W.G. Sawyer, K.J. Wahl, Mater. Res. Soc. Bull. 33, 1145 (2008).

6. A. Ovcharenko, G. Halperin, I. Etsion, Wear 264, 1043 (2008).

7. A. Ovcharenko, G. Halperin, I. Etsion, J. Tribol. 131, 011404 (2009).

8. J.M. Urueña, S.M. Hart, D.L. Hood, E.O. McGhee, S.R. Niemi, K.D. Schulze, P.P. Levings, W.G. Sawyer, A.A. Pitenis, Tribol. Lett. 66, 141 (2018).

9. A.H. Zavieh, N. Espallargas, Tribol. Int. 99, 96 (2016).

10. A. Ovcharenko, I. Etsion, J. Tribol. 131, 031602 (2009).

11. B.A. Krick, J.R. Vail, B.N.J. Persson, W.G. Sawyer, Tribol. Lett. 45, 185 (2012).

12. K.G. Rowe, A.I. Bennett, B.A. Krick, Tribol. Int. 62, 208 (2013).

13. A.I. Bennett, K.L. Harris, K.D. Schulze, J.M. Urueña, A.J. McGhee, A.A. Pitenis, M.H. Müser, T.E. Angelini, W.G. Sawyer, Tribol. Lett. 65, 134 (2017).

14. B. Weber, T. Suhina, T. Junge, L. Pastewka, A.M. Brouwer, D. Bonn, Nat. Commun. 9, 888 (2018).

15. S. Korres, T. Feser, M. Dienwiebel, Acta Mater. 60, 420 (2012).

16. J.M. Shockley, D.J. Horton, K.J. Wahl, Wear 380-381, 251 (2017).

17. S.L. Marshall, K.D. Schulze, S.M. Hart, J.M. Urueña, E.O. McGhee, A.I. Bennett, A.A. Pitenis, C.S. O'Bryan, T.E. Angelini, W.G. Sawyer, Biotribology 11, 69 (2017).

18. T.D.B. Jacobs, R.W. Carpick, Nat. Nanotechnol. 8, 108-112 (2013).

19. J.R. Felts, A.J. Oyer, S.C. Hernandez, K.E. Whitener, J.T. Robinson, S.G. Walton, and P.E. Sheehan, Nat. Commun. 6, 6467 (2015).

20. N.N. Gosvami, J.A. Bares, F. Mangolini, A.R. Konicek, D.G. Yablon, R.W. Carpick, Science 348, 102 (2015).

21. J. Wang, F. Sansoz, J. Huang, Y. Liu, S. Sun, Z. Zhang, S.X. Mao, Nat. Commun. 4, 1742 (2013).

22. L. Tian, J. Li, J. Sun, E. Ma, Z.W. Shan, Sci. Rep. 3, 1 (2013).

23. T.D.B. Jacobs, A. Martini, Appl. Mech. Rev. 69, 060802 (2017).

24. I. Szlufarska, M. Chandross, R.W. Carpick, J. Phys. D Appl. Phys. 41, 123001 (2008).

25. N. Gane, F.P. Bowden, J. Appl. Phys. 39, 1432 (1968).

26. N. Gane, Proc. R. Soc. London. A 317, 367 (1970).

27. K. Hokkirigawa, K. Kato, Z.Z. Li, Wear 123, 241 (1988).

28. W.K. Lo, J.C.H. Spence, Ultramicroscopy 48, 433 (1993).

29. J.C.H. Spence, W. Lo, M. Kuwabara, Ultramicroscopy 33, 69 (1990).

30. Y. Kondo, K. Takayanagi, Phys. Rev. Lett. 79, 3455 (1997).

31. H. Ohnishi, Y. Kondo, K. Takayanagi, Nature 395, 780 (1998).

32. I. Lahouij, F. Dassenoy, L. Knoop, J.-M. Martin, B. Vacher, Tribol. Lett. 42, 133 (2011).

33. I. Lahouij, F. Dassenoy, B. Vacher, J.-M. Martin, Tribol. Lett. 45, 131 (2011).

34. J.P. Oviedo, K.C. Santosh, N. Lu, J.G. Wang, K. Cho, R.M. Wallace, M.J. Kim, ACS Nano 9, 1543 (2015).

35. A.P. Merkle, L.D. Marks, Appl. Phys. Lett. 90, 1 (2007).

36. A.P. Merkle, A. Erdemir, O.L. Eryilmaz, J.A. Johnson, L.D. Marks, Carbon, 48, 587 (2010).

37. I.Z. Jenei, F. Dassenoy, T. Epicier, A. Khajeh, A. Martini, D. Uy, H. Ghaednia, A. Gangopadhyay, Nanotechnology 29, 85703 (2018).

38. A.A. Pitenis, J.M. Urueña, E.O. McGhee, S.M. Hart, E.R. Reale, J. Kim, K.D. Schulze, S.L. Marshall, A.I. Bennett, S.R. Niemi, T.E. Angelini, Tribol. Mater. Surf. Interfaces 11, 180 (2017).

39. A.A. Pitenis, J.M. Urueña, T.T Hormel, T. Bhattacharjee, S.R. Niemi, S.L. Marshall, S.M. Hart, K.D. Schulze, T.E. Angelini, W.G. Sawyer, Biotribology 11, 77 (2017).

40. L. Yang, Y.Y. Lua, M.V. Lee, M.R. Linford, Acc. Chem. Res. 38, 933 (2005).

41. W. Maw, F. Stevens, S.C. Langford, J.T. Dickinson, J. Appl. Phys. 92, 5103 (2002).

42. P.E. Sheehan, Chem. Phys. Lett. 410, 151 (2005).

43. B. Gotsmann, M.A. Lantz, Phys. Rev. Lett. 101, 125501 (2008). 
44. H. Bhaskaran, B. Gotsmann, A. Sebastian, U. Drechsler, M.A. Lantz, M. Despont, P. Jaroenapibal, R.W. Carpick, Y. Chen, K. Sridharan, Nat. Nanotechnol. 5, 181 (2010).

45. T.D.B. Jacobs, B. Gotsmann, M.A. Lantz, R.W. Carpick, Tribol. Lett. 39, 257 (2010).

46. R.W. Carpick, T.D.B. Jacobs, Microsc. Microanal. 20, 1542 (2014).

47. S. Raghuraman, M.B. Elinski, J.D. Batteas, J.R. Felts, Nano Lett. 17, 2111 (2017).

48. R.A. Bernal, P. Chen, J.D. Schall, J.A. Harrison, Y.R. Jeng, R.W. Carpick, Carbon 128, 267 (2018).

49. Y. Shao, T.D.B. Jacobs, Y.J. Jiang, K.T. Turner, R.W. Carpick, M.L. Falk, ACS Appl. Mater. Interfaces 9, 35333 (2017).

50. J. Zhang, H. Spikes, Tribol. Lett. 63, 24 (2016).

51. H. Spikes, Tribol. Lett. 17, 469 (2004).

52. M. Aktary, M.T. McDermott, G.A. McAlpine, Tribol. Lett. 12, 155 (2002).

53. J.M. Martin, T. Onodera, C. Minfray, F. Dassenoy, A. Miyamoto, Faraday Discuss. 156, 311 (2012).

54. C. Grossiord, J.M. Martin, T. Le Mogne, T. Palermo, Tribol. Lett. 6, 171 (1999).

55. G. Pereira, A. Lachenwitzer, D. Munoz-Paniagua, M. Kasrai, P.R. Norton, M. Abrecht, P.U.P.A. Gilbert, Tribol. Lett. 23, 109 (2006).

56. N.J. Mosey, M.H. Müser, T.K. Woo, Science 307, 1612 (2005).

57. N.N. Gosvami, J. Ma, R.W. Carpick, Tribol. Lett. 66, 154 (2018).

58. A. Akchurin, R.A. Bosman, Tribol. Lett. 65, 59 (2017).

59. A. Dorgham, P. Parsaeian, A. Azam, C. Wang, A. Morina, A. Neville, Tribol. Int. 133, 288 (2019).

60. H.S. Khare, I. Lahouij, A. Jackson, G. Feng, Z.Y. Chen, G.D. Cooper, R.W. Carpick, ACS Appl. Mater. Interfaces 10, 40335 (2018).

61. H.S. Khare, N.N. Gosvami, I. Lahouij, Z.B. Milne, J.B. McClimon, R.W. Carpick, Nano Lett. 18, 6756 (2018).

62. J.L. Gilbert, Corrosion 73, 1478 (2017).

63. W. Zhang, A.G. Carcea, R.C. Newman, Faraday Discuss. 180, 233 (2015).

64. R.J.K. Wood, Wear 376, 893 (2017).

65. D. Landolt, S. Mischler, Tribocorrosion of Passive Metals and Coatings. (Elsevier, 2011).

66. V. Swaminathan, J.L. Gilbert, J. Biomed. Mater. Res. A 101, 2602 (2013).

67. C.B. Von der Ohe, R. Johnsen, N. Espallargas, Wear 271, 2978 (2011).

68. A.H. Zavieh, N. Espallargas, Tribol. Int. 103, 368 (2016).

69. U. Malayoglu, A. Neville, Wear 255, 181 (2003).

70. Z. Wang, Y. Yan, L. Xing, Y. Su, L. Qiao, Tribol. Int. 113, 370 (2017).

71. M. Tsutsui, K. Shoji, M. Taniguchi, T. Kawai, Nano Lett. 8, 345 (2008).

72. N. Agrait, Phys. Rep. 377, 81 (2003).

73. U. Landman, W.D. Luedtke, N. Burnham, R. Colton, Science 248, 454 (1990).

74. A.J. Lockwood, K. Anantheshwara, M.S. Bobji, B.J. Inkson, Nanotechnology 22, 105703 (2011).

75. A.P. Merkle, L.D. Marks, Wear 265, 1864 (2008).

76. T. Sato, T. Ishida, L. Jalabert, H. Fujita, Nanotechnology 23, 505701 (2012).

77. Y. Lu, J.Y. Huang, C. Wang, S. Sun, J. Lou, Nat. Nanotechnol. 5, 218 (2010).

78. Y. Yang, A. Kushima, W. Han, H. Xin, J. Li, Nano Lett. 18, 2492 (2018).

79. Z. Zhang, J. Cui, B. Wang, H. Jiang, G. Chen, J. Yu, C. Lin, C. Tang, A. Hartmaier, J. Zhang, J. Luo, Nanoscale 10, 6261 (2018).

80. J. Sun, L. He, Y.C. Lo, T. Xu, H. Bi, L. Sun, Z. Zhang, S.X. Mao, J. Li, Nat. Mater. 13, 1007 (2014).

81. T. Ishida, T. Sato, S. Nabeya, K. Kakushima, H. Fujita, Jpn. J. Appl. Phys. 50, 077201 (2011).

82. S. Suresh, J. Li, Nature 456, 716 (2008).

83. P. Liu, X. Wei, S. Song, L. Wang, A. Hirata, T. Fujita, X. Han, Z. Zhang, M. Chen, Acta Mater. 165, 99 (2018).

84. W. Guo, Z. Wang, J. Li, Nano Letters 15, 6582 (2015).

85. S.B. Vishnubhotla, R. Chen, S.R. Khanal, A. Martini, T.D.B. Jacobs, Nanotechnology 30, 35704 (2019).

86. S.B. Vishnubhotla, R.M. Chen, S.R. Khanal, J. Li, E.A. Stach, A. Martini, T.D.B. Jacobs, Nanotechnology 30, 45705 (2019).

87. F. Yang, R.W. Carpick, D.J. Srolovitz, ACS Nano 11, 490 (2017).

88. F.P. Bowden, D. Tabor, Friction and lubrication of solids (Oxford University Press, 1950)

89. D.A. Rigney, M.G.S. Naylor, R. Divakar, L.K. Ives, Mater. Sci. Eng. 81, 409 (1986).

90. D.A. Hughes, N. Hansen, Phys. Rev. Lett. 87, 135503 (2001) 
91. N. Argibay, M. Chandross, S. Cheng, J.R. Michael, J. Mater. Sci. 52, 2780 (2017).

92. E. Arzt, Acta Mater. 46, 5611 (1998).

93. C. Greiner, Z. Liu, R. Schneider, L. Pastewka, P. Gumbsch, Scr. Mater. 153, 63 (2018).

94. A. Emge, S. Karthikeyan, H.J. Kim, D.A. Rigney, Wear 263, 614 (2007).

95. X. Chen, R. Schneider, P. Gumbsch, C. Greiner, Acta Mater. 161, 138 (2018).

96. C. Greiner, Z. Liu, L. Strassberger, P. Gumbsch, ACS Appl. Mater. Interfaces 8, 15809 (2016).

97. D.A. Rigney, S. Karthikeyan, Tribol. Lett. 39, 3 (2009).

98. H.M. Cao, X. Zhou, X.Y. Li, K. Lu, Tribol. Int. 115, 3 (2017).

99. K. Wolff, Z. Liu, D. Braun, J. Schneider, C. Greiner, Tribol. Int. 102, 540 (2016).

100. Z. Liu, C. Patzig, S. Selle, T. Höche, P. Gumbsch, C. Greiner, Scr. Mater. 153, 114 (2018).

101. S.J. Eder, U. Cihak-Bayr, C. Gachot, M. Rodríguez Ripoll, ACS Appl. Mater. Interfaces 10, 24288 (2018).

102. T. Sato, T. Ishida, L. Jalabert, H. Fujita, Tribol. Online 6, 226 (2011).

103. B. Bhushan, Fundamentals of Tribology and Bridging the Gap between the Macro-and Micro-/Nanoscales (Springer Science and Business Media, Netherlands, 2012). 